Dermatologische Zeitschrift. 1917;24:I-V

\title{
Contents, Vol. 24, 1917
}

Inhalts -Verzeichnis.

Original-Arbeiten. $\quad$ S $\beta 1 \mathrm{t} \beta$

Almkvist, Job., Die experimentelle Quecksilberstoma

titis des Kaninchens. (Hierzu Taf. I-V) 1

$\mathrm{B}$ a c h , $\Gamma$. W., Ein Beitrag zuBruc k's serochemischer Re-

aktion bei Syphilis 204

Bardach,K., Über die Salpetersäurereaktion auf Syphilis

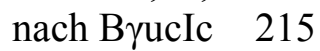

Callomon, Fritz, HauttuberkuJose und Tuberkulide

bei Heeresangehörigen

716

Chable, Robert E., Über Kerion Celsi und lichenoides

Exanthem bei Audouinischer Mikrosporie 20

Cronquist. C, Zur Entstehungsweise deГ Warzen . . . 559

Fönss, AageL., Die Fokalreaktion nach der Tuber kulin-

injektion beim Lupus vulgaris

533

Frieboes, W., Über ein Haemato-Lymphangiom des

Halses und der Zunge. (Hierzu Taf. VI-VIII). . . .120

- $\quad-$, Multiples, idiopatbisch.es Lymphosarcoma cutis, Sar-

comatosis cutis Spiegler und sarkoide Tumoren. (Hierzu

Taf. XIV-XV) 257

- $\quad-$, Haarpinselbildung auf der sonst vollkommen haarlosen

Kopfhaut eines kongenital-syphüitischen Kindes . . . .477

, Vaseline- und Teerschäd.igung der unbedeckten und

belichteten Körperhaut

641

Gold, E., Anatomische Untersuchung eines Falles von

Herpes zoster 100

Goldmann, R., Pigmentveränderungen der Haut und

Haare und Alopecie infolge von Verletzungen des Zentral-

nervensystems .

359

Ha $x$ th a u s e n ,H,Pili annulati 298

Herxheimer, K., und Nathan, E., Über Sensibili-

sierung der Haut durch Carboneol gegenüber Sonnenlicht

und eine dadurch bedingte Dermatitis Solaris

385

, Über glyzerinfreie Schüttelmixturen: Cremor

Zinci und Lac Zinci 449

- IV -

Seit $\beta$

Hesse, Max, Elephantiasis des Penis 480

Hoffmann, E., Über Neurinome der Haut (Neuro- 
fibrome Recklinghausens, unausgereifte Neurome Kyrles)

und ihre Bedeutung für die Naevusfrage 295

J a li n e 1, Über die Spirochaeta pallida bei der progressiven

Paralyse. (Hierzu Taf. XVII-XVIII) 604

Kauf mann- Wolf, M., Sind Tabiker und Paralytiker

noch infektios? $\quad 390$

Kissmeyer, A., Psoriasis bullosa. (Hierzu Taf. XVI) . 397 Kyrle, J., Beitrag zur Kenntnis der

multiplen, unausge-

reiften Hautneurome. (Hierzu Taf. XIII)

Lindenheim, H, Zur Kenntnis der systematisierten

Naevi. (Hierzu Taf. IX-XII) 144

L o e b , H., Zum Nachweis der Gonokokken . . 646

Lomholt, S., Ein Fall von Akrodermatitis chronica atro-

phicans (Herxheimer) mit sekundären Infiltrationen und

Ulzerationsbildung 485

Meirowsky, Das Problem der Pigment bildung im Lichte

der neuen Forschungen Blochs und seiner Mitarbeiter . 705 Michael, M., Beiträge zur Kasuistik

und Differ entialdia-

gnose seltener frühluetischer und gonorrhoischer Kompli-

kationen 406

Muschter, ÜberdieVerwendungder PonndorfschenTuber -

kulinbehandlung in der Dermatologie 451

Peddersen, M. Br $33 / 8, n$, Ein Beitrag zum Studium der

Pathogenese der sekundären Tricbophytide ...... 731

Per ut z, A., Über Dermographismus, cine Quecksilber-

reaktion der Haut bei Syphilitikern 165

P o 11 a n d, R., Zur Klinik und Ätiologie der Impetigo gan-

graenosa Kreibichs 286

, Zur Klinik der Hautveränderungen bei Pseudoleukämie

und bei Mycosis fungoides 321

Pontoppidan ,B., Über das Vorkommen von Allgemein-

erscheinungen, insl \&gt; esondere Blutveränderungen bei

Lichen ruber planus 341

P u 1 v e r m a c h e r, L., Zur Prage des Spätikterus nach Sal-

varsan 577, 648

$\mathrm{R}$ u e t e , A. E., Beitrag zur Behandlung der Syphilis durch

die kom.binierte Neosalvarsan-Hg-Salicyl-Kur

$\mathrm{S}$ c i p a 1 , N., Atrophia cutis m, aculosa unter dem Bilde einer

Dermographia selecta persistens 471

- V-

$\overline{\text { Seite }}$

S utter, E., Weitere Beiträge zur Lehre von der Immunität

und Überempfindlichkeit bei Trichophytieerkrankungen 65

Zimmern, F., Zur Ätiologie des idiopathischen Herpes

zoster 32

Gesellschaftsberichte. 
Berliner Dermatologische Gesellschaft. Sitzung vom 5. De-

zember 1916217

Sitzung, vom 13. März 1917422

"Sitzung, vom 8. Mai $1917 \quad 620$

ïagung der Südwestdeutscben Dermatologischen Gesellschaft 239 -- der Beutschen

Gesellschaft für Geschlechtskrankheiten 493

Dermatologentag in Bonn 678

Royal society of Medicine. Dermatolog. Section 687

Periodische Literatur 45, 115, 177, 244, 304, 367, 435, 495,

$564,630,701$

Buchanzeigen 320, 382, 572

Tagesnachriehten und Personalien 64, 128, 192, 256, 320,

$448,512,576,640,749$

Nekrologe.

KarlPosselt 64

Edward Welander 240

Caesar Boeck 305

Therapeutische Notiz 448

Sachregister 750

Namenregister 759 